Trivent Publishing

(C) The Authors, 2015

Available online at http://trivent-publishing.eu/

Philosophy, Communication, Media sciences Series

Volume Saint Gerard of Cenad: Tradition and Innovation

\title{
Der Widerstand gegen Rechtsextremismus: Die antitotalitäre Haltung der Banater Eliten in der Zwischenkriegszeit
}

\author{
Mihai A. Panu \\ West-Universität, Temeswar, Rumänien, mihai.panu@e-uvt.ro
}

\begin{abstract}
Soon after World War I, the entire European continent experienced major socio-political transformations. The emerging structural economic and political imbalances triggered an unprecedented radicalization of various societal relations and attitudes which eventually reached a culminating point during World War II.

This paper investigates the manifestations of National Socialism in interwar Banat and the key role played by the Catholic Church as an ideological counteracting force. In this highly controversial and disproportionate relation not only the pure facts or end results represent a historiographical stake but also the key public figures which somehow managed to influence or change the course of history. In the interwar Banat, a decisive public role in the struggle of containing the impact of the Nazi ideology was played by the catholic bishop Dr. Augustin Pacha.
\end{abstract}

Keywords: NS-ideology; Banat; political extremism; Augustin Pacha; propaganda.

This work was co-financed by the European Social Fund through Sectoral Operational Programme Human Resources Development 2007-2013; project number POSDRU/159/1.5/S/140863, Competitive Researchers in Europe in the Field of Humanities and Socio-Economic Sciences. A Multi-regional Research Network.

This is an Open Access article distributed in accordance with the Creative Commons Attribution Non Commercial (CC-BYNC-ND 4.0) license, which permits others to copy or share the article, provided original work is properly cited and that this is not done for commercial purposes. Users may not remix, transform, or build upon the material and may not distribute the modified material (http://creativecommons.org/licenses/by-nc/4.0/)

DOI: $10.22618 /$ TP.PCMS.20151.176013 


\section{Einleitung}

Im Kontext der zunehmenden Radikalisierung des sozial-politischen Systems in Europa der Zwischenkriegszeit, gewann der ideologische Faktor in allen Bereichen des öffentlichen Lebens stark an Bedeutung. Die unerwarteten Konsequenzen des Ersten Weltkrieges und die systemischen Schwächen des Parlamentarismus, verursachten in vielen Staaten Europas eine rasche Entwicklung der radikalen politischen Phänomene. Auf der geopolitischen Bühne Europas, zwei wichtige ideologische Akteure beanspruchten die Vormacht: National-Sozialismus und Kommunismus. Aufgrund ihrer revolutionären weltanschaulichen Programme, versuchten beide Regimetypen ihre Interessensphären auszudehnen bis zu dem Punk wo der generalisierte Konflikt unvermeidlich war. $\mathrm{Zu}$ Beginn des Zweiten Weltkrieges war Europa ideologisch schon stark polarisiert. Mit dem Zerfall der politischen gemäßigten Mitte, begann ein kontinuierliches Eindringen der beiden extremen Polen des politischen Spektrums in die öffentlichen Sphäre der meisten europäischen Staaten. In diesem Prozess der strategischen ideologischen Eroberung möglichst vieler Gesellschaftssegmente, die effizienteste Methode und zugleich, das Hauptinstrument zur Verwirklichung dieser ideologisch motivierten Zielvorgabe, war die Propaganda.

Das Hauptziel der vorliegenden Arbeit besteht darin, die Erscheinungsformen der nationalsozialistischen Propaganda im Banat der Zwischenkriegszeit zu analysieren und die Rolle der römischkatholischen Kirche als ideologischer Gegenfaktor in diesem besonderen Kontext zu untersuchen.

Die nazional-sozialistische Propaganda entwickelte sich im Banat in der obenerwähnten Zeitspanne, besonders nach der Machteroberung Hitlers anfangs 1933. In Rumänien die NaziPropagandakampagnen erfolgten unter besonderer Kontrolle der zentralen Naziverwaltung und hatten als Ziel vorwiegend eine Neukonfiguration der ideologischen Orientierungen und Präferenzen, sowohl der verschiedenen gesellschaftlichen Klassen als auch der rumänischen politischen Eliten. Diese, von den National-Sozialisten instrumentalisierte Propaganda, fand auf rumänischem Gebiet, in vielen Fällen, günstige Bedingungen, aufgrund einiger präexistenten Verhältnisse. Diese favorisierende Faktoren widerspiegeln die politische und sozio-kulturelle Entwicklung Großrumäniens nach 1918. Für die rumänische politische Elite, die Verwaltung eines multiethnischen und multikonfessionellen sozialen Korpus erwies sich als problematisch von Anfang an. Die Verwirklichung in 1918 des „Nationaltraumes“ aller Rumänen wurde kurz danach eine politische Herausforderung für die unvorbereitete und stark fragmentierte rumänische politische Klasse. Rumänien war nicht in der Lage die Loyalität der verschiedenen nationalen Minderheiten zu gewinnen und auch keine Kohesion des gesamten nationalen Korpus zu sichern.

Das Fehlen eines Gemeinschaftssinnes in Großrumänien verursachte in der ersten Phase eine Art, Demütigungsgefühl sowohl bei der ethnischen Mehrheit, deren Erwartungen, aufgrund mangelhafter politischen Partizipation und unterentwickelter materiellen Lage (im Vergleich zu den Minderheiten, vor allem Juden und Deutsche), ${ }^{1}$ kurzfristig nicht erfüllbar waren, als auch bei den Minderheiten, die politisch unterrepräsentiert waren und über ein, vom rumänischen Staat ausgegebenen, Minderheitengesetzt nicht verfügten. ${ }^{2}$

Der rumänische Zwischenkriekszeitnationalismus ging zum Teil aus diesen sozio-politischen Gegebenheiten hervor. Als Folge der politischen Unfähigkeit Großrumäniens die Interessen aller ethno-kulturellen Gruppen zentralistisch zu vertreten, und somit eine authentische nationale Identität zu fördern, verschlechterten sich die sozialen Beziehungen vor allem zwischen den zwei bereits polarisierten Kategorien: Mehrheit und Minderheiten. Diese Polarisierung wurde von den neu entstandenen politischen Gruppen rechts-extremer Orientierung, sofort ideologisch instrumentalisiert.

Die rumänischen Rechts-Extremisten gewannen langsam an Bedeutung auf der politischen Bühne und shafften auch eine überraschende institutionelle Entwicklung in allen Regionen des Landes.

\footnotetext{
${ }^{1}$ Sugar Peter: Naţionalismul est-european în secolul al XX-lea, Bucureşti, Curtea Veche, 2002, S. 236.

${ }^{2}$ Maner Hans Christian: Parlamentarismul în România 1930-1940, Bucuresti, Editura Enciclopedica, 2004 , S. 305.
} 
M. A. Panu 141

Solche extremistische Organisationen ${ }^{3}$ hatten einen raschen Aufstieg nach der Entstehung Großrumäniens und erreichten ihre Blütezeit gegen Ende der 30er Jahren. Die Krise des Parlamentarismus ermöglichte die soziale Legitimation dieser radikalen Organisationen. Man konnte leicht diese Tatsache in der wachsenden Anzahl der Anhänger erkennen. Die Entwicklung des rumänischen Nationalismus in der Zwischenkriegszeit hatte einen direkten Anschlag in allen Schichten der Gesellschft. Die Anhängerschaft dieser Organisationen bestand nicht nur aus einfachen, leicht beeinflussbaren Personen sondern auch aus Individuuen mit einem gewissen Bildungsniveau.

Im rumänischen Banat die Erscheinungsformen des Nationalismus waren frühzeitig erkennbar. Das soziopolitische Profil der Banater Gesellschaft war traditionell pluralistisch und aus diesem Grund, als inkompatibel mit Radikalismen jedwelcher Art betrachtet, aber auch diese Region der vorausgesetzten Toleranz, blieb von den Auswirkungen des politischen Extremismus nicht verschont.

Beginnend mit dem Aufstieg des einheimischen Nationalismus, verschlechterte sich kontinuierlich die soziale Lage im Banat. Die ethnokulturellen Antagonismen wurden von der extremistischen Propaganda stark betont und wiederholt im öffentlichen Diskurs reproduziert. Die Auswirkungen der Propaganda waren auch in der Banater Gesellschaft frühzeitig erkennbar. In der lokalen Banater Presse, z.B. in der von L.A.N.C finanzierten, „Svastica Banatului,““ einen heftigen Antisemitismus wurde systematisch schon in dem ersten Jahrzent nach der Enstehung Großrumäniens propagiert. Später, als die Nazional-Sozialisten in Deutschland die politische Macht beanspruchten, jubelte die radikale Banater Presse und zeigte die ideologische Verwandschaft des deutschen und rumänischen Nationalismus auf:

„Heil Hitler. Hervorragender Erfolg der deutschen Antisemiten bei den allgemeinen Wahlen am 14. September. Ungefähr 6.5 Millionen Stimmen auf der Liste mit dem Hakenkreuz. Das Judentum in der ganzen Welt schreit, wie aus dem Schlangenmaul. Da nähert sich das Urteil des internationalen Judentums. (...) Der bewundernswerten antisemitischen Partei, die sich Nationalsozialistisch nennt (mit einem Programm nahezu identisch mit dem des „Bundes der Nationalen Christlichen Verteidigung“ in Rumänien) unter der Führung des deutschen Helden Adolf Hitler ist es gelungen, die zweite Position (nach der Zahl der Abgeordneten) im politischen System Deutschlands zu erobern.(...) Nach dem Erfolg von Hitler sind wir berechtigt unsere Überzeugung zu äußern: die Zukunft gehört uns! Nur wir sind in der Lage das verarmte Europa zu retten. (A.d.Ü). ${ }^{\text {"5 }}$

Die ideologischen Gemeinsamkeiten der beiden radikalen politischen Strömungen in Deutschland und Rumänien, bereiteten den Weg für das Eindringen der Nazi-Propaganda in das Banat. In diesem Kontext, der gemeinsame Nenner, welcher die entscheidende Rolle spielte, sowohl in der deutschen als auch in der rumänischen Gesellschaft, war den Antisemitismus. Unter diesen komplexen geopolitischen Bedingungen, wenige gesellschaftliche Akteure waren in der Lage einen konstruktiven Widerstand gegen den propagandistischen Druck der Extremisten zu leisten.

Im rumänischen Banat einer der wichtigsten Akteuren, der systematisch versuchte die Auswirkungen des Totalitarismus zu bekämpfen, war die Römisch Katholische Kirche. Unter der Führung des damaligen Bischofs dr. Augustin Pacha, erwies sich die katholische Diözese in Temeswar als einer wahrhaften Oppositionsfaktor sowohl des rumänischen Rechtsextremismus als auch des deutschen Nationalsozialismus. Pacha, der als Bischof der Temeswarer Diözese am 16 Oktober $1930^{6}$ ernannt wurde, setzte sich im Laufe seiner Amtszeit als gesellschaftlicher und politischer Akteur durch, besonders im Kontext der zunehmenden antiklerikalen Politiken des, im Banat wirkenden Nationalsozialismus.

\footnotetext{
${ }^{3}$ Es handelt sich vorwiegend um: L.A.N.C (Liga Apărării Naţional Creştine/Bund der Nationalen Christlichen Verteidigung, A.d.Ü) oder die später entstandene Eiserne Garde.

${ }^{4}$ Banater Swastika (A.d.Ü).

${ }^{5}$ Svastica Banatului, Jahrgang IV, Nr. 31, 15 September 1930.

${ }^{6}$ Kräuter Franz: Erinnerungen an Bischof Pacha. Ein Stück Banater Heimatgeschichte, Bukarest, ADZ Verlag, 1995, S. 109.
} 
Die später umstritten gewordene Persönlichkeit des Bischofs (besonders aus ideologischer Sicht) wurde in den rumänischen politischen Kreise als besonders prolematisch betrachtet, wenn man z.B. bedenkt dass der anfangs 1934 stattgefundene Besuch Pachas bei Adolf Hitler, heftige Aufregung in der rumänischen Öffentlichkeit erregte. Die Rolle Pachas als Exponent der klerikalen Autorität im Banat kann nicht untersucht werden ohne dass die systemischen Beziehungen zwischen Politik und Religion in Betracht gezogen werden. Die Relevanz dieser Beziehungen im Kontext der Entstehung und Entwicklung totalitärer Regime ist äußerst wichtig. Aus geopolitischer Sicht, die Nationalsozialsten betrachteten die Katholische Kirche von Anfang an, als eine Art ideologischer Konkurrent. Diese Rivalität zeigte sich später als die politischen Entscheidungsträger des NaziRegimes versuchten, die traditionelle Position der Katholischen Kirche im gesellschaftlichen Bewusstsein zu untergraben. Sowohl die Kirche als auch die Politik beanspruchen (selbstverständlich in verschiedenen Formen) eine gewisse Autorität auf gesellschaftlichen Niveau.

Die von der katholischen Kirche ausgeübte Autoritätsform ähnelt sich in vielen Hinsichten der typischen Machtausübung der politischen Regime, (wie der deutsche Theoretiker Carl Schmitt behauptete): „The political power of Catholicism rests neither on economic nor on military means but rather on the absolute realization of authority." Die Rivalität zwischen Kirche und Politik ergibt sich eben aus diesen übereinstimmenden Machtpositionen. Als totalitäre Ideologie beanspruchte der Nationalsozialismus die totale Macht d.h. in allen Bereichen des öffentlichen Lebens. Eine Teilung dieser Macht mit anderen Akteuren (z.B. die Kirche) war aus ideologischer Perspektive nicht akzeptabel weil die Existenz anderer Machtforen, für den Nazionalsozialismus einfach eine Entlegitimierungsgefahr darstellte.

Im rumänischen Banat, die Römisch-Katholische Kirche spielte eine wichtige soziale Rolle vor allem in den Reihen der Banater-Schwaben. Unter der Führung dr. Pachas verwaltete die Temeswarer Diözese mehrere konfessionelle Schuleinrichtungen sowie verschiedene kirchenahen Organisationen wie zum Beispiel „Der Deutsch-Katholische Jugendbund” oder „Die Katholische Deutsche Mädchenkränze.“8 ${ }^{\text {(8) }}$ Diese, von der katholischen Kirche kontrollierten Organisationen, sind schnell ein strategisches Ziel für die Nationalsozialisten geworden, vor allem weil die Jugendlichen die Haupmitgliedschaft darstellten. Solche Organisationen waren (zusammen mit den konfessionellen Schulen) das Hauptziel der Propagandakampagnien weil eine der wichtigsten Strategien der Nationalsozialisten war dafür bestimmt möglichst viele junge Personen ideologisch anzuziehen und somit eine effiziente Indoktrination der jungeren Generationen zu ermöglichen. Für die politischen Entscheidungsträger des Nazi-Regimes, die strategische Relevanz der Katholischen Kirche im rumänischen Banat ergab sich aus dem Potential dieser Institution ein traditionsgemässes, gesellschaftlich wünschenswertes Erziehungssystem anzubieten. Eine, von der Kirche erzogene Gemeinschaft, benimmt sich in der Regel skeptisch gegenüber Politik und Ideologie und kann aus diesem Grund schwer politisch indoktriniert werden. Um die Loyalität der Menschen zu gewinnen musste der Nationalsozialismus propagandistisch agieren um die Position der Kirche zu schwächen und ihre erzieherische soziale Rolle zu beenden. Diese Tatsache stellte im Banat den Schwerpunkt in den Auseinandersetzungen der Katholischen Kirche mit dem Nationalsozialismus.

\section{Die antitotalitären Strategien der klerikalen Eliten im Banat}

Nach der Enstehung Grossrumäniens (1918) nahm die Zahl der deutschen Schulen kontinuierlich zu. Die rumänische Regierung versuchte die Loyalität der deutschen Bevölkerung zu gewinnen indem den Zugang zum deutschsprachigen Unterricht für alle rumänische Staatsbürger deutscher Abstammung garantiert war. Ebenfalls versuchte man durch diese Massnahmen das ethno-kulturelle Bewusstsein der deuschen Minderheit nach jahrelanger Madjarisierung zur Zeit der Donaumonarchie, zu erwecken.

\footnotetext{
${ }^{7}$ Schmitt Carl: Roman Catholicism and Political Form, Westport, Greenwood Press, 1996, S.18.

(Die politische Macht des Katholizismus beruht weder auf wirtschaftlichen noch auf militärischen Mitteln, sondern vielmehr auf der absoluten Erkenntnis der Autorität. A.d.Ü).

${ }^{8}$ Der Ruf, Temeswar, Januar 1937, S. 4, Archivum Dioecesanum Timisoarense.
} 
M. A. Panu

Die Neugestaltung einer kulturellen Identität der Banater Schwaben gehörte zu den Leitlinien der rumänischen Politik in der Zwischenkriegszeit. Neben den, vom Staat verwalteten Schulen, entwickelten sich systematisch auch die konfessionellen Schulen:

„Im Banat war die Zahl der katholisch-konfessionellen Schulen gewachsen, auch in mehreren Mädchenmittelschulen des Notre-Dame Ordens war allmählich die deutsche Unterrichtssprache eingeführt worden und die kath. deutsche Lehrerbildungsanstalt stellte neben dem Realgymnasium die zweite tragende Säule des Banater deutschen Schulwesens dar. Ebenso konnte die enge Zusammenarbeit zwischen der nun gefestigten neuen schwäbischen Volksführung und der führenden Geistlichkeit als Beweis dafür gelten, dass im Banat auch die katholische Kirche Beschützerin des angestammten Volkstums sein kann.“9

In diesem besonders günstigen politischen Kontext, die Banater Schwaben hatten die Möglichkeit ihre kulturelle und Bildungsinfrastruktur zu erweitern. Die Katholische Kirche und die von dieser Kirche kontrollierten lokalen politischen Organisationen, spielten in diesem Prozess eine entscheidende Rolle. Das institutionelle Gewicht der Katholischen Kirche ermöglichte eine konstante Vertretung der Volksgruppeinteressen sowohl auf lokalem als auch auf zentralem politischen Niveau. Die klerikalen Kreise förderten kontinuierlich die Gründung verschiedener Schuleinrichtungen und erzielten eine möglichst erweiterte Schulautonomie der deutschen Minderheit. Eine kurze Übersicht über das Banater schwäbische Schulwesen zwischen 1934 - 1936, zeigt die folgende Situation auf: ${ }^{10}$

- 16 konfessionelle mittlere und höhere Schulen mit rund 2000 Schülern und Schülerinnenund 140 deutschen Lehrkräften (Die „Banatia“ hatte nun die frühere Stellung des „Realgymnasiums“ eingenommen und war zum wichtigsten Kulturzentrum des Banater Deutschtums geworden)

- Etwa 65 konfessionelle Volksschulen mit rund 9700 Kindern und 165 deutschen Lehrern.

- Ein staatliches Knabenlyzeum mit rund 500 Schülern und 12 deutschen Lehrkräften.

- Und etwa 115 staatliche Volksschulen mit rund 21500 Kindern und 300 deutschen Lehrkräften.

Diese stark entwickelte Bildusngsinfrastruktur der Banater Schwaben blieb von den ideologischen Tendenzen der damaligen Zeit nicht verschont. Der geopolitische Druck der NS-Ideologie hatte konkrete Auswirkungen in Rumänien besonders nach 1933 aber die ersten Erscheinungsformen des Nationalsozialismus im Banat waren früher erkennbar. Als Vermittler dieser Ideologie gelten nicht nur die pan-europäischen radikalen politischen Strömungen die eine institutionell-strukturierte Verbreitung der NS-Hauptideen ermöglichten sondern auch einzelne Personen die als ideologische Vektoren agierten. Rumänien war von den europäischen politischen Weltanschauungen nicht komplett isoliert. Man konnte schon in den ersten Jahren nach der Großunion von 1918 eine gewisse Empfänglichkeit der Bevölkerung für Nationalsozialismus und Kommunismus (betrachtet al ideologische Zwillinge der Zwischenkriegszeit) feststellen. Unter diesen Umständen, das ethnokulturelle Profil der deutschen Minderheit in Rumänien spielte eine entscheidende Rolle für die politische Orientierung vieler Rumäniendeutschen. Die Verinnerlichung der nationalsozialistischen Denkstrukturen vor allem durch die Jugendlichen erfolgte aus diesem Grund quasi automatisch. In diesem Fall handelt es sich nicht um eine Art kultureller Determinismus obwohl die kulturelle Identität und die ethno-kulturellen Kompatibilitäten vorgegeben sind. Die Empfänglichkeit eines Teils der deutschen Bevölkerung in Rumänien für Nationalsozialismus war kulturell ermöglicht (z.B. das Fehlen der sprachlichen Barrieren die das Verstehen der Nazi-Propaganda ermöglichte) aber nicht kulturell bedingt. Dieser Aspekt ist entscheidend für eine geeignete Untersuchung der politischen Geschichte der deutschen Minderheit in Rumänien. Die Tatsache dass die Rumäniendeutschen zum gesamteuropäischen deutschen Kulturkreis gehörten und ihre ethno-kulturelle Identität vorwiegend

\footnotetext{
9 Hügel Kaspar: Abriss der Geschichte des Donauschwäbischen Schulwesens, München, Verlag des Südostdeutschen Kulturwerks, 1957, S. 18.

${ }^{10}$ Ibidem, S. 18-19.
} 
M. A. Panu

dadurch konstruiert war, verursachte eine frühzeitige ungehinderte ideologische Beeinflussung dieser Minderheit.

Im Banat die Vorreiter des Nationalsozialismus agierten als überzeugte politische Aktivisten indem sie ein revolutionäres sozial-politisches Programm förderten.

„Der Wegbereiter der nationalsozialistischen Erneuerung im Banat ist Oberst Karl von Möller. Seit 1922 hielt er die Verbindung zur NSDAP aufrecht . Er kam jedoch erst 1931 zum Aufbau der ersten nationalsozialistischen Gruppen. Die ersten entstanden in Hatzfeld (Möller und Peter Maurusz), Neu-Arad (Franz Stoss und Peter Bernath), Perjamosch (Rudolph Ferch und Franz Adam Minnich) und Temeschburg (Geisa Buding und Peter Schmidt)." "11

Die Verbreitung der NS-Ideen in Rumänien erfolgte in vielen Fällen durch die Beteiligung der Jugendorganisationen. Solche Organisationen (z.B. der antiklerikale „Jungschwäbische Klub im Banat" ${ }^{\text {12 }}$ oder die „Wandervogel") $)^{13}$ hatten einen gemeinsamen Nenner und zwar den romantischrevolutionären Anspruch, die sozio-politischen Verhältnisse der damaligen Zeit neuzudefiniren. Die meisten politisch orientierten Organisationen der Banater Schwaben übernahmen die ideologischen Leitlinien der, am 22 Mai 1932 von Fritz Fabritius gegründeten N.S.D.R - „Nationalsozialistische Selbsthilfebewegung der Deutschen in Rumänien." "Das Eindringen des Nationalsozialismus in die Banater Gesellschaft erfolgte schrittweise und erreichte einen Höhepunkt zur Zeit des Zweiten Weltkrieges als die von Berlin kontrollierte Organisation „DviR - Deutsche Volksgruppe in Rumänien" von der rumänischen Regierung als Person des öffentlichen Rechts annerkant wurde:

„(...) am 20 November wurde die DViR durch Antonescu im Sinne der Karlsburger Beschluesse von 1918 in einem Volksgruppen-Dekret zu einer Person des öffentlichen Rechts erklärt."

Die Entwicklung der NS-Ideologie im Banat war nicht ausschliesslich die Konsequenz verschiedener Einstellungen oder Prefärenzbildungsprozessen innerhalb der Banater deutschen Minderheit sondern auch das Resultat systemischer Ungleichgewichte in der rumänischen sozial-politischen Sphäre. Die Radikalisierung des politischen Systems und die Krise des Parlamentarismus verursachten eine zunehmende Polarisierung der gesamten rumänischen Gesellschaft. In Folge dessen die sozialen politischen Orientierungen und Präferenzen richteten sich allmählich nach dem bestehenden ideologischen Angebot.

Die wichtigste institutionelle Erscheinungsform des Nationalsozialismus im Banat war die DViR. Diese, in September $1940^{16}$ gegründete Organisation, funktionierte aufgrund ihrer, vom rumänischen Staat garantierte Autonomie, wie eine Art Staat im Staate. Unter der Führung Andreas Schmidts, ein am 24 Mai 1912 in Donnersmark ${ }^{17}$ (Siebenbürgen) geborener SS-Offizier, ${ }^{18}$ der an der Spitze der

11 Hockl Nik. Hans: Das deutsche Banat. Seine geschichtlich-politische Entwicklung und Aufgabe, Temeschburg, Buchdruckerei Anwender\&Sohn, 1940, S. 57.

${ }^{12}$ Ibidem, S.54.

${ }^{13}$ Ibidem, S. 57. Diese Organisation gab es im Banat schon im Jahre 1926. Unter der Führung von Nikolaus Hans Hockl und mit Beteiligung verschiedener ideologisch stark motivierten Rumäniendeutschen (z. B. Paul Kindl, Peter Maurusz, Peter Lindacher, Sepp Komantschek, Sepp Schmidt, Stefi Heinz) wurde die Banater Wandervogelorganisation ein Instrument der NS-Propaganda. (A.d.V.)

${ }^{14}$ Ibidem.

${ }^{15}$ Milata Paul: Zwischen Hitler, Stalin und Antonescu. Rumäniendeutsche in der Waffen-SS, 2. Auflage, Köln, Böhlau, 2009, S. 77.

Cf. Böhm Johann: Die Deutschen in Rumänien und das Dritte Reich. 1933-1940, Frankfurt am Main, Peter Lang Verlang, 1999, S. 313.

${ }^{16}$ Milata Paul: Zwischen Hitler, Stalin und Antonescu. Rumäniendeutsche in der Waffen-SS, 2. Auflage, Köln, Böhlau, 2009, S. 77.

${ }^{17}$ Böhm Johann: Nationalsozialistische Indoktrination der Deutschen in Rumänien. 1932-1944, Frankfurt am Main, Peter Lang Verlag, 2008, S. 109. 
M. A. Panu

DViR, direkt vom Leiter der Volksdeutschen Mittelstelle (VoMi-Chef SS- Obergruppenfuehrer Werner Lorenz) ernannt wurde, ${ }^{19}$ begann die ideologische Einmischung in die inneren Angelegenheiten der Katholischen Kirche im Banat.

Unter diesen Umstände begann die Temeswarer Diözese langsam die strategische Initiative gegenüber den ideologischen Druck der NS-Ideologie zu verlieren. Nach wiederholten Anforderungen der DViR, musste die Katholische Kirche im Banat an alle Jugendverbände und Frauenorganisationen verzichten. Dies geschah offiziell durch das Rundschreiben nr. 1537/1941 am 6 Mai 1941. ${ }^{20}$ Ein Jahr später, im Mai 1942 wurden auch die, von der Katholischen Kirche verwalteten konfessionellen Schulen, von der Deutschen Ethnischen Gruppe in Rumänien übernommen. Diese Übernahme geschah nich nur mit der alleinigen Zustimmung Bischof Pachas sondern auch mit der formellen Einwilligung Vatikans (durch die diplomatische Vertretung des Heiligen Stuhls in Bukarest):

„Als einige Wochen später der Päpstliche Nuntius in Bukarest einem Vertreter der Volksgruppenführung gegenüber die Versicherung der Zustimmung gegeben hatte, wurde es von Bischof Pacha und dem Volksgruppenführer Andreas Schmidt am 16 März 1942 unterzeichnet, am 6 Mai 1942 durch Unterrichtsminister Petrovici bestätigt und am 26 Mai 1942 im Amtsblatt veröffentlicht." ${ }^{21}$

In diesem äußerst ungünstigen ideologische Kontext, versuchte Augustin Pacha die spezifischen Interessen des Katholizismus $\mathrm{zu}$ vertreten. Aufgrund einer zunehmend ungünstig gewordenen, institutionellen Beziehung zwischen Religion und Politik (besser gesagt zwischen Katholizismus und Nationalsozialismus), da diese Beziehung die damaligen strategischen Machtbeziehungen widerspiegelte und somit eine Machtbeziehung zu Gunsten des politischen Faktors war, musste Pacha seine Kommunikationsstrategien anpassen und die Diplomatie als geeignetes Handlungsinstrument in Betracht ziehen. Die antiklerikale Propaganda und alle, gegen die Kirche gerichteten, Maßnahmen der Nationalsozialisten, konnten nicht einfach nur durch die traditionellen, vorwiegend von der symbolischen Macht geprägten, Handlungsinstrumente der Kirche bekämpft werden. Pacha versuchte zunehmend eine politische Rolle in der Öffentlichkeit zu spielen, in der Hoffnung dass eine grundsätzlich politisch und ideologisch definierte gesellschaftliche Ordnung kann nur durch gleichartigen d.h. politisch zielorientierte Handlungsstrategien beeinflusst werden. Die Anpassungsfähigkeit Pachas an die damaligen ideologischen Verhälnisse und seine wiederholten Versuche, die Politik zu Gunsten der Kirche zu beeinflussen, sind, wenn man seine Tätigkeit auch aus politischer Perspektive analysiert, leicht zu erkennen. Ein Beispiel dafür ist die Initiative Pachas, hochdiplomatisch zu agieren und sogar Adolf Hitler, bezüglich der zunehmenden Bedrohung der Banater kirchlichen Ordnung durch die NS-Ideologie, anzusprechen. ${ }^{22}$

Augustin Pacha hatte persönliche Kontakte mit Hitler schon im Februar 1934 aufgenommen. Das Treffen zwischen Hitler und Pacha bleibt heutzutage immer noch ein umstrittenes Thema weil diese Begegnung besonders von der rumänischen Öffentlichkeit als ein Zeichen der endgültigen nationalsozialistischen Orientirung des Banater Bischofs betrachtet wurde. Pacha versucht den Kontext seines Treffens mit Hitler in einem unveröffentlichen, im Archiv der Temeswarer katholischen Diözese aufbewahrten $\operatorname{Text}^{23}$ zu erklären:

\footnotetext{
${ }^{18}$ BArch, NS 19/1077, S. 9.

${ }^{19}$ Milata Paul: Zwischen Hitler, Stalin und Antonescu. Rumäniendeutsche in der Waffen-SS, 2. Auflage, Köln, Böhlau, 2009, S. 77-78.

${ }^{20}$ Archivum Dioecesanum Timisoarense, ungeordnet.

${ }^{21}$ Hügel Kaspar: Das Banater deutsche Schulwesen in Rumänien von 1918 bis 1944, München, Verlag des Südostdeutschen Kulturwerkes, 1968, S. 87.

${ }^{22}$ Es handelt sich um einen Brief, der von Pacha am 31 Dezember 1940, an die Führerkanzlei in Berlin geschickt wurde. Cf. PA AA, R93351/189, S. 198.

${ }^{23}$ Archivum Dioecesanum Timisoarense, Manuskript.

Es handelt sich um ein ungefähr 100 Seiten langes Manuskript, mit dem Titel „Zur Geschichte der Deutschen in heutigen Rumänien“ das im Arhiv der katholischen Diözese in Temeswar aufbewahr ist. Der Name des Autors
} 
M. A. Panu

„(...)Ermutigt durch den Eindruck auf einige Nazis, habe ich mich entschlossen Hitler zu begegnen und ihm über unsere Sorgen zu berichten. Hitler hörte mir sehr höflich zu und schien von meiner Präsentation beeindruckt zu sein. Aber am nächsten Tag die deutsche Depeschenagentur veröffentlichte eine Erklärung und berichtete dass ich dem Führer Adolf Hitler, die Würdigungs der Banater Schwaben präsentiert hätte. Diese ungenaue und später rektifizierte Nachricht, hatte kein anderes Ziel als meine Entehrung in Bukarest zu Gunsten und wahrscheinlich nach der Anforderung meiner Nazi-Gegner. Ich habe es begriffen, dass in unserem Kampf gegen den Nationalsozialismus nicht mehr, wie in der Vergangenheit, auf die Bekümmertheit der Berliner kulturellen Interessen für unser Volk zählen können. Berlin beschäftigte sich offiziell nicht mehr mit den Interessen des deutschen Volkes sondern mit den Interessen des Nationalsozialismus. Wer kein überzeugter Nationalsozialist war, wurde automatisch als Feind betrachtet.( A.d.Ü).‘24

Pachas antitotalitäres Verhalten ist, aufgrund seiner öffentlich angenommenen, politisch-orientierten Rolle, nicht für alle Historiker und Zeitzeugen deutlich genug. Ihm wurde eine gewisse Mitschuld im Prozess der nationalsozialistischen Indoktrination verschiedener sozialen Schichten im Banat, vorgeworfen:

„Die katholische Kirche bildete keine ideologische Front gegen die Erneuerungsbewegung. Bischof Pacha nahm von Anfang an eine neutrale Haltung ein und behielt diese auch gegenüber der Volksgruppenführung Schmidt.. ${ }^{25}$

Die Beziehungen zwischen der Katholischen Kirche im Banat und den verschiedenen, vom Dritten Reich kontrollierten NS-Organisationen waren notwendigerweise Machtbeziehungen und die Nazis, aufgrund ihrer geopolitisch überlegenen Position, ergriffen in den meisten Fällen die Initiative. Dem Bischof war es bewusst dass die Katholische Kirche gegen den Nationalsozialismus keine ideologische Front bilden kann weil die Vertreter dieser Kirche als Kleriker, im Kampf gegen eine totalitäre Ideologie keine ideologieartige Diskursstrategien verwenden können.

Die Bekämpfung der NS-Ideologie durch eine Art kirchliche Gegen-Ideologie, würde automatisch eine Entlegitimisierung der Katholischen Kirche bedeuten. Augustin Pacha versuchte dieser Entlegitimierungseffekt $\mathrm{zu}$ vermeiden indem er seine Handlungsstrategien gegen den Nationalsozialismus vorwiegend als politische und diplomatische (und nicht als ideologische) Aktionen einsetzte. Pacha ging davon aus dass eine ideologische Auseinandersetzung der Kirche mit dem Dritten Reich keine realistische Strategie ist, weil die Kirche nicht über legitime, ideologieähnliche Diskursinstrumente verfügt. Außerdem, die Kirche hatte nicht das gleiche Propagandapotential und geopolitische Überlegenheit wie die totalitäre Ideologie des Nationalsozialismus. Unter diesen ungünstigen Umständen, vesuchte Pacha die sozialen Auswirkungen der NS-Ideologie in der Banater Gesellschaft einzudämmen, vor allem durch nichtideologische Maßnahmen.

\section{Abschließende Bemerkungen}

Die Amtszeit Augustin Pachas stimmte mit dem Aufstieg der extremistischen politischen Bewegungen überein. Die ideologisch-religiösen Auseinandersetzungen im Banat der Zwischenkriegszeit hatten vorwiegend einen strategischen Charakter. Die Katholische Kirche versuchte schrittweise und zielgerichtet nicht unbedingt einen totalen Widerstand gegen die NS-Ideologie zu leisten, (weil eine solche Strategie unrealistisch und sogar gefährlich wäre), sondern vielmehr die Auswirkungen dieser Ideologie auf gesellschaftlichen Niveau zu bändigen. Das strategische Ziel des Dritten Reiches bestand darin, die von der Katholische Kirche kontrollierten Organisationen (darunter auch die konfessionellen

wurde vom Deckblatt entfernt aber aufgrund verschiedener stilistischen und biographischen Hinweise, kann man davon ausgehen dass das Manuskript höchstwahrscheinlich dem Bischof Augustin Pacha gehört.(A.d.V.).

${ }^{24}$ Archivum Dioecesanum Timisoarense, Manuskript, S. 10-11.

${ }^{25}$ Hartl Hans: Das Schicksal des Deutschtums in Rumänien, Würzburg, Holzner, 1958, S. 44-45. 
M. A. Panu

Schulen) zu übernehmen und somit die Mitglieder dieser Organisationen (vor allem Jugendliche) von dem Einfluss der Kirche zu trennen. Das menschliche Kapital spielte für die Indoktrinationsstrategien der Nationalsozialisten eine entscheidende Rolle. Besonders die Jugendlichen, deren Emfänglichkeit gegenüber revolutionären Weltanschauungen frühzeitig ausgeprägt war, bildeten die strittige und ideologisch wünschenswerte soziale Kategorie. In 1934 existierten im rumänischen Banat die folgenden, von der Katholischen Kirche verwalteten Organisationen: ${ }^{26}$

- Deutsch - Katholischer Jugendbund: ungefähr 5000 Mitglieder

- Der Katholisch-Deutsche Frauenverein und Mädchenkranz: ungefähr 7000 Mitglieder

- Arbeitsgemeinschaft der Jungpriester: ungefähr 40 Mitglieder

Wenn man bedenkt dass in diesen Organisationen die Mitgliedschaft vorwiegend aus Jugendlichen bestand, wird auch der propagandistische Druck des Dritten Reiches leicht erklärbar. Der totalitäre Anspruch der NS-Ideologie erforderte die Loyalitätsgewinnung möglichst breiter Gesellschaftsschichten. Sowohl die Politik als auch die Religion (betrachtet als generische institutionalisierte Suprastrukturen) benötigen eine kontrollierbare Anhängerschaft um sich zu legitimieren.

Wenn man davon ausgeht dass das (politische) Verhalten der Gesellschaft von der institutionellen Suprastruktur bestimmt ist d.h. wenn eine radikale Ideologie zur Suprastruktur wird und das institutionelle Design eines Staates (und somit das alltägliche Leben) prägt, die gesellschaftlichen Prozessen (Einstellungen gegenüber ethnokulturellen Pluralismus, politische Präferenzen etc.) werden ebenfalls ideologisch geprägt. Dieser Struktur-Akteur Determinismus hat eine besondere Relevanz für die aktuelle Geschichtsschreibung. Das daraus resultierende Erklärungsmuster ist aus mehreren Perspektiven am besten geeignet um den sozio-politischen Kontext im Banat der Zwischenkriegszeit zu erklären.

\section{Bibliographie}

Böhm, Johann. Die Deutschen in Rumänien und das Dritte Reich. 1933-1940. Frankfurt am Main: Peter Lang Verlang, 1999.

. Nationalsozialistische Indoktrination der Deutschen in Rumänien. 1932-1944. Frankfurt am Main: Peter Lang Verlag, 2008.

Hockl, Nik. Hans. Das deutsche Banat. Seine geschichtlich-politische Entwicklung und Aufgabe. Temeschburg: Buchdruckerei Anwender\&Sohn, 1940.

Hügel, Kaspar. Abriss der Geschichte des Donauschwäbischen Schulwesens. München: Verlag des Südostdeutschen Kulturwerks, 1957.

. Das Banater deutsche Schulwesen in Rumänien von 1918 bis 1944. München,: Verlag des Südostdeutschen Kulturwerkes, 1968.

Kräuter, Franz. Erinnerungen an Bischof Pacha. Ein Stück Banater Heimatgeschichte. Bukarest: ADZ Verlag, 1995, S. 109.

Maner, Hans Christian. Parlamentarismul în România 1930-1940. Bukarest: Editura Enciclopedica, 2004.

Milata, Paul. Zwischen Hitler, Stalin und Antonescu. Rumäniendeutsche in der Waffen-SS, 2. Auflage. Köln: Böhlau, 2009.

Schmitt, Carl. Roman Catholicism and Political Form. Westport: Greenwood Press, 1996.

Sugar, Peter. Nationalismul est-european în secolul al XX-lea. [Der osteuropäische Nationalismus im 20 Jahrhundert], Bukarest: Curtea Veche, 2002.

\footnotetext{
${ }^{26}$ PA AA, Bukarest 136/2587, Dossier I.A-3: Vermittlungsmission Dr. Scherers.
} 


\section{Archivquellen}

Der Ruf, Temeswar, Januar 1937, S. 4, Archivum Dioecesanum Timisoarense.

Svastica Banatului, Jahrgang IV, Nr. 31, 15 September 1930, Biblioteca Judeţeană Timiş, Secţia Documentar-Periodice.

***, Zur Geschichte der Deutschen in heutigen Rumänien, Archivum Dioecesanum Timisoarense, Fond Politic, ungeordnet.

BArch, NS 19/1077, Andreas Schmidt, Volksgruppenführer der Deutschen Volksgruppe in Rumänien.- Differenzen mit dem SD in Rumänien, Verwendung bei Kriegsende in einem Einsatzkommando, Das Bundesarchiv, Berlin Lichterfelde.

PA AA, R93351/189, Reichs- und Volksdeutsche in Rumänien, I. Das politische Archiv des Auswärtigen Amtes, Berlin Mitte.

PA AA, Bukarest 136/2587, Römisch-Katholische Kirche, Dossier I.A-3: Vermittlungsmission Dr. Scherers, Das politische Archiv des Auswärtigen Amtes, Berlin Mitte. 